\title{
Simultaneous presentation of sarcoidosis and acute myeloid leukaemia: Predisposition to pulmonary haemorrhage
}

\author{
B Williams, A Francis, S Durrant
}

\begin{abstract}
A case of coexistent acute myeloid leukaemia and sarcoidosis is reported. This was complicated by recurrent pulmonary haemorrhage during reinduction and consolidation chemotherapy. A review of published papers on malignancy and sarcoidosis, in particular acute leukaemia is given. The outcome of most cases of acute leukaemia and sarcoidosis is poor with respiratory complications a frequent cause of death in this group. It is proposed that modifications to treatment to avoid pulmonary toxicity and maintenance of platelet counts above $40 \times 10^{9} / 1$ are warranted to reduce the risk of this complication.
\end{abstract}

( $\mathcal{~ C l i n ~ P a t h o l ~ 1 9 9 4 ; 4 7 : 6 7 2 - 6 7 3 ) ~}$

\section{Case report}

A 23 year old man presented for investigation of recurrent mouth ulcers over several months, splenomegaly, and neutropenia. The blood count at presentation showed neutrophils $0.4 \times 10^{9} / 1$, haemoglobin $104 \mathrm{~g} / \mathrm{l}$, and

Department of Haematology, Royal Brisbane Hospital, Herston, Queensland, Australia 4029 B Williams A Francis S Durrant Correspondence to: Dr S Durrant

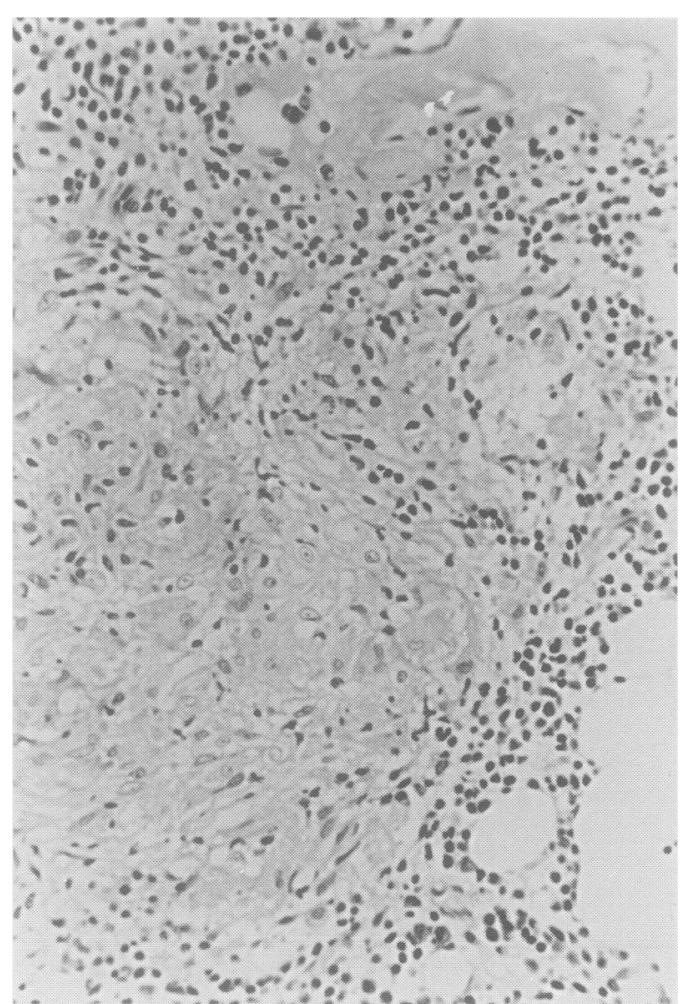

Marrow trephine section demonstrating non-caseating granuloma. a normal platelet count. There was no relevant history of illness, infection, or drug or toxin exposure. A bone marrow aspirate showed a mildly hypocellular marrow with $35 \%$ myeloperoxidase positive blasts. Trephine sections showed numerous noncaseating granulomata (figure) with no evidence of organisms on Ziehl Neelson, periodic acid Schiff, and Grocott stains. Cultures of the marrow for fungi and bacteria, including mycobacteria, were negative. A chest $x$ ray picture and computed tomography showed fine reticulonodular shadowing, and a subsequent transbronchial lung biopsy specimen revealed non-caseating granulomata. These findings in addition to a substantially increased serum angiotensin converting enzyme (90 U/1-normal range 13-40 U/1) enabled sarcoidosis to be diagnosed. $\mathrm{He}$ was given prednisone for sarcoidosis and subsequently started leukaemia induction treatment with idarubicin and cytosine. Marrow recovery, particularly neutrophil count, was extremely slow, preventing consolidation therapy. Four months later relapse was confirmed on bone marrow examination prompted by the appearance of blasts in the peripheral blood. No granulomata were evident at this time. Reinduction and first consolidation using idarubicin and high dose cytosine were complicated by episodes of cough, dyspnoea, fever and hypoxia with pulmonary infiltrates evident on chest $x$ ray picture. Pulmonary haemorrhage was confirmed on transbronchial lung biopsy specimens. Substantial thrombocytopenia $\left(<20 \times 10^{9} / 1\right)$ was present on each occasion. These episodes were managed with platelet transfusion, antibiotics and oxygen. No pulmonary complications occurred during second consolidation with the same drug doses, but platelet counts were actively maintained at $>40 \times 10^{9} / 1$. He went into remission. Steroids were stopped seven months after diagnosis as there was no clinical or biochemical evidence of active sarcoidosis. Further relapse of leukaemia occurred four months later and the patient succumbed to non-pulmonary sepsis.

\section{Discussion}

The association of sarcoidosis and malignancy - both haematological and non-haematological-is well documented. ${ }^{1-10}$ In most cases the diagnosis of one disease is followed chronologically by the other with few cases of coexisting disease. Reports of sarcoidosis preceding malignancy are the most common, 
with patients with chronic active sarcoidosis having a fivefold increase in the risk compared with the general population. ${ }^{12}$ Lymphoma, particularly Hodgkin's disease, is the commonest malignant tumour in this group. ${ }^{1-3}$ It has been proposed that immune dysregulation and impaired $T$ cell response, due to depletion of circulating $\mathrm{T}$ helper cells, may increase susceptibility to oncogenic viruses. ${ }^{12}$

Sarcoidosis following malignancy is less common, with variable times elapsing between the diagnosis of the two diseases. The reasons for the development of sarcoidosis are unknown. It has been postulated that chemotherapy and radiotherapy may have an evolutionary role, but the occurrence of the two entities by chance alone cannot be excluded. ${ }^{24}$ Sarcoidosis occurring simultaneously with malignancy has only rarely been reported.

The association of acute leukaemia and sarcoidosis is rare, with eight published cases reported. ${ }^{4-10}$ Of these, six cases of leukaemia followed the diagnosis of sarcoidosis by 3-7 years and two were concurrent. One patient had previously received cytotoxic treatment with methotrexate ${ }^{4}$ and two had been treated with ionising radiation. ${ }^{3}$ The possibility that leukaemia represented a secondary malignancy in these cases must be considered. Explanations for the association between leukaemia and sarcoidosis have included impaired immune surveillance as a result of the sarcoidosis predisposing to the development of leukaemia. ${ }^{8}$ In cases where the leukaemia and sarcoidosis occur concurrently, it has been suggested that the granulomata represent an immunological response to tumour antigens. ${ }^{10}$

The outcome in most cases of leukaemia is poor with four deaths occurring before treatment could be started, one death early in the course of treatment, and one death due to relapse a year after complete remission was achieved. Of the remaining patients, one achieved remission after one course of treatment but no information on follow up was given and one received allogeneic marrow transplantation in remission and was alive in continuing remission three years later.

Respiratory complications are common in this group, occurring in four of the reported patients. Three patients presented with pulmonary infiltrates, one thought to be due to infection, ${ }^{8}$ one thought to be due to a leukaemic infiltrate, ${ }^{9}$ and the cause of the third not postulated. ${ }^{10}$ However, no histological diagnosis was given and in at least one patient no biopsy or necropsy was performed. The remaining patient died of respiratory failure, but no information on the cause was provided. Three patients died before the start of induction chemotherapy, with the remaining patient dying within 48 hours of starting treatment. The case we report developed major respiratory complications in response to treatment which were confirmed on two occasions to be due to pulmonary haemorrhage. The risk of pulmonary complications seems to be increased in patients with sarcoidosis and leukaemia. Modifications to treatment, including avoidance of drugs with lung toxicity, should be considered. Maintenance of platelet counts at levels greater than $40 \times 10^{9} / 1$ is also recommended to reduce the risk of pulmonary haemorrhage in these patients.

1 Brinker $\mathrm{H}$. The sarcoidosis-lymphoma syndrome. $\mathrm{Br} \mathcal{F}$ Cancer 1986;54:467-73.

2 Brinker $H$. Solid tumours preceding or following sarcoidosis. Med Ped Oncol 1987;15:82-8.

3 Maloisel F, Oberling F. Acute myeloid leukaemia and sarcoidosis. F Roy Soc Med 1992;85:58-9.

4 Hermann C, Anderson E, Videback A. Acute myeloblastic leukaemia in sarcoidosis treated with methotrexate. Scand $\mathcal{F}$ Haematol 1980;24:234-6.

5 Hatake K, Aoyagi N, Takaku F. Acute myelomonocytic leukaemia following remission of sarcoidosis. Oncology 1983;40:200-1.

6 Nordenson I, Bjermer L, Holmgren G, Hornstein P, Wahlin A. $t(6 ; 9)$ in bone marrow cells in two patients with sarcoidosis and acute myeloid leukaemia. Cancer wenet Cytogenet 1989;38:297-300.

7 Hoffbrand V. Sarcoidosis and leukaemia. Sarcoidosis 1989;6:87.

8 Murphy EA, Murphy JA, Sturrock RD. Sarcoidosis and acute leukaemia. F Roy Soc Med 1991;84:368-9.

9 Rab SM, Kazmi KA, Vahidy F. Sarcoidosis and acute monoblastic leukaemia. Sarcoidosis 1990;7:72-3.

10 Reich JM. Acute myeloblastic leukaemia: implications for treatment. Cancer 1985;55:366-9. 